University of Nebraska - Lincoln

DigitalCommons@University of Nebraska - Lincoln

1986

\title{
Angular and Energy Dependence of Cross Sections for Ejection of Electrons from Water Vapor. I. 50-2000-eV Electron Impact
}

\author{
M. A. Bolorizadeh \\ University of Nebraska - Lincoln \\ M. Eugene Rudd \\ University of Nebraska - Lincoln, erudd@unl.edu
}

Follow this and additional works at: https://digitalcommons.unl.edu/physicsrudd

Part of the Physics Commons

Bolorizadeh, M. A. and Rudd, M. Eugene, "Angular and Energy Dependence of Cross Sections for Ejection of Electrons from Water Vapor. I. 50-2000-eV Electron Impact" (1986). M. Eugene Rudd Publications. 10. https://digitalcommons.unl.edu/physicsrudd/10

This Article is brought to you for free and open access by the Research Papers in Physics and Astronomy at DigitalCommons@University of Nebraska - Lincoln. It has been accepted for inclusion in M. Eugene Rudd Publications by an authorized administrator of DigitalCommons@University of Nebraska - Lincoln. 


\title{
Angular and energy dependence of cross sections for ejection of electrons from water vapor. I. 50-2000-eV electron impact
}

\author{
M. A. Bolorizadeh* and M. E. Rudd \\ University of Nebraska-Lincoln, Lincoln, Nebraska 68588-0111
}

(Received 20 May 1985)

\begin{abstract}
We have measured the angular and energy distribution of the absolute cross sections for ejection of electrons from water vapor by electrons incident at $50-2000 \mathrm{eV}$. The angular range was $15^{\circ}$ to $150^{\circ}$ and the range of secondary electron energies was from $2 \mathrm{eV}$ to an energy equal to the primary energy minus the first ionization potential. Electron energies were analyzed with a parallel-plate electrostatic analyzer with a resolution of $1.1 \%$. Generally good agreement is obtained with the data of Opal et al. [J. Chem. Phys. 55, 4100 (1971)] at $500 \mathrm{eV}$ except at the extremes of their angular range. Our energy distributions are compared to various binary-encounter-model calculations.
\end{abstract}

\section{INTRODUCTION}

Doubly differential cross sections (DDCS) in which the angular and energy distribution of electrons resulting from electron-atom or electron-molecule collisions have been measured for a number of different gas targets by several groups. Water vapor measurements, normalized to elastic scattering cross sections, were made by Opal et al. ${ }^{1}$ over an angular range of $30^{\circ}$ to $150^{\circ}$ but only at 500 $\mathrm{eV}$. The same angular range was covered by Vroom and Palmer ${ }^{2}$ using primary energies from $1-10 \mathrm{keV}$. Vroom's measurements were normalized at each electron energy to the total ionization cross section data of Schutten et al. ${ }^{3}$ $\mathrm{Oda}^{4}$ has reported measurements over the range of $15^{\circ}$ to $148^{\circ}$ at two primary energies 500 and $1000 \mathrm{eV}$. These measurements have been normalized to elastic scattering cross sections of Bromberg. ${ }^{5}$

While the data of Opal et al. ${ }^{1}$ integrated over angle are generally believed to be accurate, their angular distributions have been brought into question ${ }^{6}$ especially at the extremes of their range. Furthermore, the energy range of their detected electrons only extended to about half the primary energy. In the work of Vroom and Palmer ${ }^{2}$ a spherical retardation analyzer was used with the DDCS being obtained by differentiating the yield curve. Besides the inherent disadvantages of this type of system, the angular resolution was rather poor. No data at all are available below $500 \mathrm{eV}$ primary energy and all of the previous DDCS data have been normalized to other measurements.

The present series of measurements was carried out at 14 different primary energies from 50 to $2000 \mathrm{eV}$. The angles were $15^{\circ}, 20^{\circ}$, and every $20^{\circ}$ from $30^{\circ}$ to $150^{\circ}$. Cross sections were integrated over angle to yield singly differential cross sections (SDCS) and over both angle and ejection energy to give the total ionization cross sections. Our cross sections are absolute since all quantities needed to calculate the cross sections such as the primary beam current, the detector efficiency, geometrical quantities, and the target gas pressure, were measured.

\section{EXPERIMENTAL PROCEDURE}

The basic apparatus has been described previously ${ }^{6,7}$ so only a brief description will be given except where changes have been made.

An electron gun with an einzel lens focusing element provided the primary electron beam. This was modified in three ways from the one previously described. The indirectly heated oxide-coated cathode was replaced by a directly heated thoria-coated iridium filament. Since only the tip of the filament was thoriated, the energy distribution of the beam had a full width at half-maximum (FWHM) of only $0.43 \mathrm{eV}$ for a $12-\mathrm{V}$ filament voltage. The second modification was to improve the insulation between the gun elements to allow the energy range to be extended to $2000 \mathrm{~V}$. The third improvement was to rebuild the housing of the gun to provide a suppressor after the last defining aperture. This prevented the low-energy electrons produced at the edges of the defining apertures from entering the collision region. A shield after the suppressor prevented the suppressor's field from entering the collision region and also stopped most of the electrons elastically scattered from the aperture.

A biased, shielded Faraday cup was used to monitor the beam for most of the measurements. In spite of the attention to collimation of the beam mentioned above, it was found that a small fraction of the beam struck the shield around the entrance to the Faraday cup causing spurious low-energy electrons. For some runs where this was a problem, the Faraday cup was swung out of the beam path entirely. The beam current was sufficiently stable that it could be read before a run was made and checked again at the end of the run. In this case, timing rather than beam integration was used. Magnetic fields in the collision and detection regions were reduced to below 5 $m G$ by a magnetic shield just inside the vacuum chamber.

A parallel-plate electrostatic analyzer with an energy resolution of $1.1 \%$ was used with a channeltron detector. A secondary Faraday cup formerly attached to the back 
of the analyzer was removed to prevent spurious electron reflections. This eliminated a troublesome "bump" in the energy distribution at about $\frac{1}{3}$ the primary energy due to scattered primary electrons. To provide an easily measured detection geometry, no preacceleration of the secondary electrons was used.

A new control and data acquisition system was built using a microcomputer. This stepped the voltage on the electrostatic analyzer, counted the amplified pulses from the detector, monitored the pressure reading from the capacitance manometer, monitored the temperature, calculated the correction factors, and combined the measured quantities to give the cross sections. Corrections were made for the absorption of both primary and secondary electrons in the target gas.

The water vapor target was derived from triply distilled and deionized water contained in a stainless-steel cell. This was purged of air and any other dissolved gases by freezing with liquid nitrogen, pumping, and then warming. This process, repeated three times, was sufficient to purify the water as checked by a quadrupole gas analyzer. The freeze-pump-thaw procedure was carried out once more each day to ensure purity of the sample. The water vapor was admitted to the chamber through a needle valve. Typical target pressures were 0.5 to $1 \mathrm{~m}$ Torr while the background pressure with no target gas was $(3-4) \times 10^{-7}$ Torr.

To measure the channeltron efficiency, the primary beam current into the cup was first measured. Then, keeping the gun conditions fixed, the gun was rotated to aim directly into the analyzer. Using the channeltron as a Faraday cup, the analyzer voltage was swept over the peak and the area under the profile measured. From this data the transmission of the analyzer was determined. This was done for a range of beam currents down to about $0.5 \mathrm{nA}$. Then the beam current was lowered to about 1 pA and the process repeated using the channeltron in the counting mode. A comparison of the effective transmission for the two cases yielded an efficiency of $0.94 \pm 0.06$ at a primary energy of $190 \mathrm{eV}$. The relative efficiency at other energies was then determined by varying the cone potential on the channeltron keeping all other parameters constant. The efficiency was found to fall to 0.76 at 2000 eV.

Uncertainties in our measurement arise from the determination of target gas pressure (5\%) and detector efficiency $(6 \%)$. Adding in additional small sources of error, we have a basic uncertainty of $9 \%$ in the DDCS. Small values of the cross sections, however, may have large errors due to uncorrected backgrounds and statistical errors in the low count rate. Cross sections for electrons of energies below about $15 \mathrm{eV}$ are subject to two additional errors of unknown size. Spurious electrons from surfaces make the cross sections too large, while stray electric and magnetic fields degrade the effective analyzer transmission and reduce the measured cross sections. While these two errors have opposite effects, it is unlikely that they would cancel out. We estimate that the uncertainty rises below $15 \mathrm{eV}$ to $50 \%$ at $2 \mathrm{eV}$. The effect of spurious electrons is most severe at the smallest angle, $15^{\circ}$, and the uncertainty of the measurements there may be on the order of $50 \%$ at most energies and greater at low energies.

The uncertainty in the singly differential cross section (SDCS) is slightly larger due to possible errors in the integration over angle. We estimate it to be $12 \%$ above 15 $\mathrm{eV}$ rising to $50 \%$ at $2 \mathrm{eV}$. There is an additional error in the integration over secondary electron energy caused by the uncertainty in extrapolating the cross sections to zero energy. Therefore, we estimate the uncertainty in the total ionization cross sections to be $15 \%$.

\section{EXPERIMENTAL RESULTS}

\section{A. Doubly differential cross sections}

The doubly differential cross sections show the same basic features for all angles as shown in Fig. 1 for $1500 \mathrm{eV}$ primary electrons. The cross sections fall rapidly as the secondary energy $W$ is increased reaching a minimum and then rising again as $W$ approaches $T-B_{1}$ where $T$ is the primary energy and $B_{1}$ is the binding energy of the outer subshell of the target. The rise, of course, is due mainly to inelastically scattered primary electrons which we cannot distinguish from secondary electrons. These scattered electrons come predominantly at small angles.

The structure seen just below $500 \mathrm{eV}$ is due to Auger electrons from $1 s$ vacancies in oxygen. Since these Auger electrons are ejected mostly isotropically, the peak shows up most strongly in the backward direction where the continuum background is the smaller. Structure is also noted between 900 and $1000 \mathrm{eV}$ which is due to primaries which have lost energy in ejecting $1 s$ electrons from oxygen. Some of these structures display the asymmetric Fano line shape resulting from interference with continuum electrons of the same energy.

Figure 2 shows the angular distributions of various energies of ejected electrons for $500-\mathrm{eV}$ primaries. At the higher energies our results are in quite good agreement

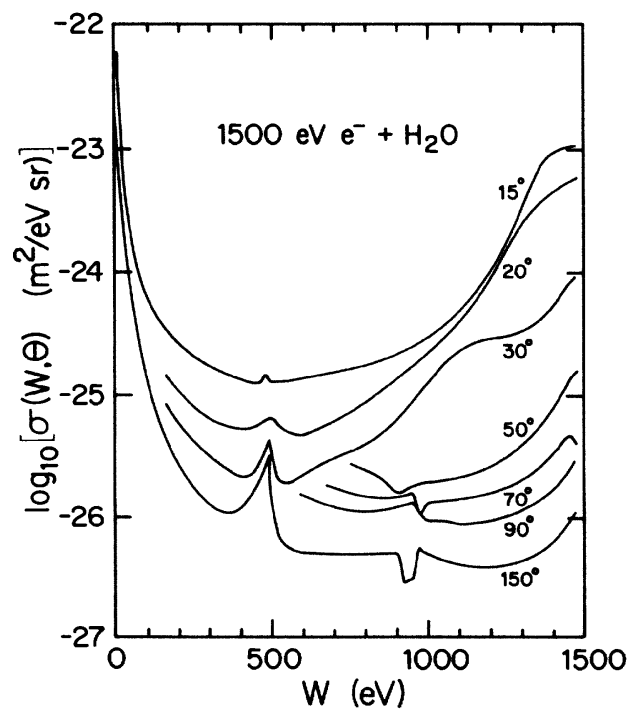

FIG. 1. Energy distributions of electrons at various angles from $1500-\mathrm{eV}$ collisions of electrons with water vapor. 


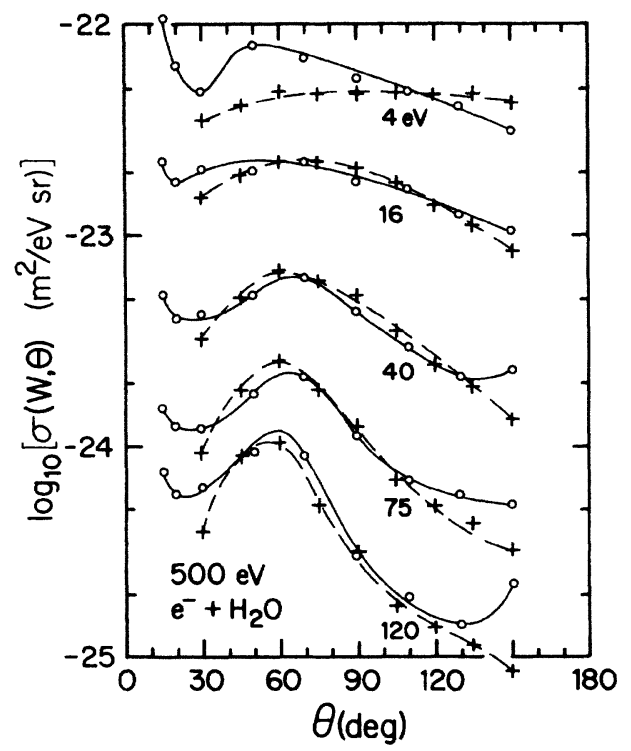

FIG. 2. Angular distributions of electrons of various energies from $500-\mathrm{eV}$ collisions of electrons with water vapor. Present data, O; data of Opal et al. (Ref. 1), +.

with those of Opal et al. ${ }^{1}$ except for the extreme ends of their angular range where, as has been noted, ${ }^{6,7}$ their cross sections are probably too low.

At most energies our angular distributions show a peak in the forward direction. This forward peak was previ- ously seen in helium by $\mathrm{Oda}^{4}$ and by Ehrhardt et al. ${ }^{8}$ Tahira and $\mathrm{Oda}^{9}$ interpreted it in terms of a binary encounter calculation in which the exchange term contributed a strong forward component. Later, however, Oda and Nishimura ${ }^{10}$ found that the sharp rise disappeared when they improved their electron-gun optics and added a retarding potential between their analyzer and detector. This seemed to indicate that the sharp rise at small angles is due to spurious low-energy electrons which manage to get through the analyzer even when it is set to a higher energy.

In our apparatus a suppressor built into the electron gun effectively prevented low-energy electrons from the gun apertures from entering the collision region. Removing the Faraday cup and its shield from the back of the analyzer eliminated another source of low-energy secondary electrons. Furthermore, a slot in the back plate allowed high-energy electrons to pass through the analyzer without striking the back plate where they could produce spurious low-energy electrons. In spite of these precautions, we still observed the peak in the forward direction. However, when we added a grounded or negatively biased grid between the analyzer and detector as in Oda and Nishimura's apparatus, we also found that the sharp rise in the forward direction disappeared. Therefore, the abnormally large values of cross sections at $15^{\circ}$ seen in Fig. 2 are most likely spurious. This is estimated to cause at most a 3\% error in the cross sections integrated over angle because of the $\sin \theta$ factor in the integration.

TABLE I. Singly differential cross sections $\left(10^{-23} \mathrm{~m}^{2} / \mathrm{eV}\right)$.

\begin{tabular}{|c|c|c|c|c|c|c|c|}
\hline \multicolumn{8}{|c|}{ Projectile energy $(\mathrm{eV})$} \\
\hline Energy & 50 & 100 & 200 & 300 & 500 & 1000 & 2000 \\
\hline 2 & 240 & 250 & 210 & 160 & 110 & 65 & 34 \\
\hline 4 & 170 & 160 & 130 & 110 & 70 & 41 & 21 \\
\hline 6 & 130 & 120 & 100 & 85 & 57 & 30 & 16 \\
\hline 8 & 110 & 93 & 80 & 66 & 44 & 24 & 13 \\
\hline 10 & 92 & 74 & 69 & 53 & 36 & 20 & 11 \\
\hline 14 & 71 & 50 & 47 & 38 & 26 & 14 & 8.4 \\
\hline 20 & 66 & 32 & 28 & 22 & 16 & 9.0 & 5.3 \\
\hline 25 & 81 & 24 & 20 & 16 & 12 & 6.5 & 3.9 \\
\hline 34 & 140 & 18 & 12 & 9.6 & 6.9 & 4.1 & 2.4 \\
\hline 40 & & 17 & 9.7 & 7.0 & 5.1 & 3.3 & 1.8 \\
\hline 48 & & 18 & 6.9 & 5.2 & 3.8 & 2.3 & 1.3 \\
\hline 56 & & 22 & 5.4 & 3.9 & 2.7 & 1.7 & 0.95 \\
\hline 70 & & 36 & 4.3 & 2.5 & 1.8 & 1.1 & 0.63 \\
\hline 100 & & & 4.0 & 1.4 & 0.87 & 0.55 & 0.32 \\
\hline 150 & & & 11 & 1.2 & 0.40 & 0.25 & 0.15 \\
\hline 200 & & & & 2.5 & 0.28 & 0.16 & 0.091 \\
\hline 240 & & & & 5.6 & 0.26 & 0.11 & 0.065 \\
\hline 300 & & & & & 0.38 & 0.074 & 0.042 \\
\hline 400 & & & & & 0.14 & 0.056 & 0.025 \\
\hline 500 & & & & & & 0.050 & 0.025 \\
\hline 700 & & & & & & 0.095 & 0.013 \\
\hline 900 & & & & & & & 0.011 \\
\hline 1200 & & & & & & & 0.0099 \\
\hline 1500 & & & & & & & 0.021 \\
\hline 1900 & & & & & & & 0.14 \\
\hline
\end{tabular}




\section{B. Singly differential cross sections}

By numerical integration using the equation

$$
\sigma(W)=2 \pi \int \sigma(W, \theta) \sin \theta d \theta
$$

we obtained the SDCS which represent the energy spectrum of all electrons from the collisions. The results of this integration for selected primary and secondary energies are given in Table I. Figures 3 and 4 also show the DDCS for three different primary energies. In Fig. 3 our results are compared with those of Opal et al. ${ }^{1}$ and with those of Vroom and Palmer. ${ }^{2}$ The agreement with the former is quite good over the middle range of energies $(10-100 \mathrm{eV})$. Our data are slightly higher at the extremes of Opal's energy range, the discrepancy being $22 \%$ at 4 $\mathrm{eV}$ and about $50 \%$ at $200 \mathrm{eV}$. Our data are in good agreement with those of Vroom at energies below $6 \mathrm{eV}$ but are higher by an increasing amount as the energy increases. At $200 \mathrm{eV}$ our results are a factor of 2.5 higher.

In Figs. 3 and 4 we also compare our results with three binary encounter model calculations. The simplest of these is the Mott formula which is basically the classical Thomson equation modified for electron impact to take account of the indistinguishability of scattered and ejected electrons. The form given by $\mathrm{Kim}^{11}$ can be slightly rewritten as

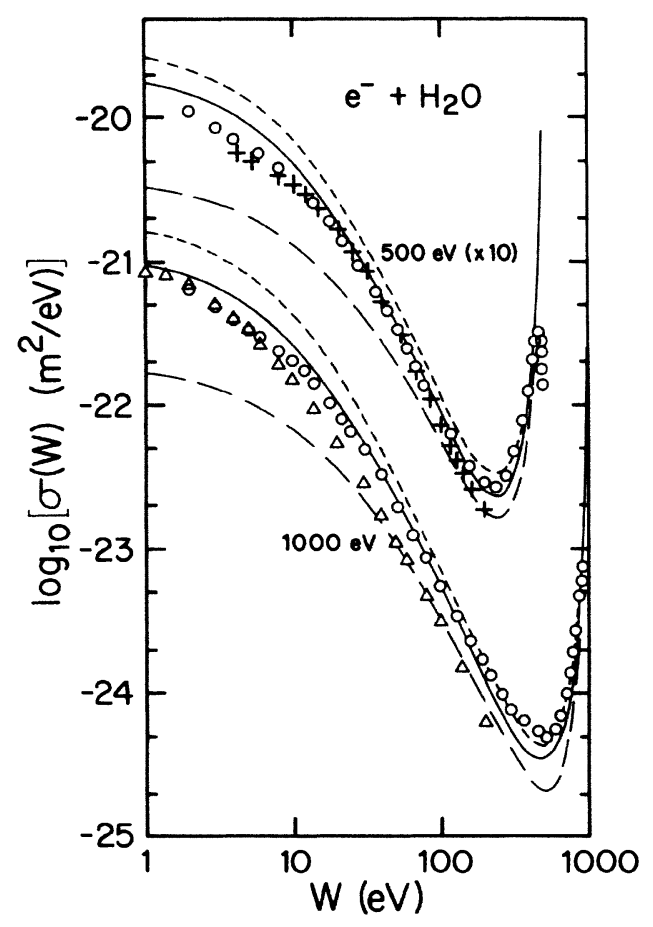

FIG. 3. Energy distributions, integrated over all angles, of electrons from 500 - and $1000-\mathrm{eV}$ collisions of electrons with water vapor. Present data, $O$; data of Vroom and Palmer (Ref. 2), $\triangle$; data of Opal et al. (Ref. 1), + . Theoretical calculations shown by the lines are for the Mott equation, long dashes; Gryzinski's equation (Ref. 14), short dashes; Vriens equation (Refs. 12 and 13), solid line.

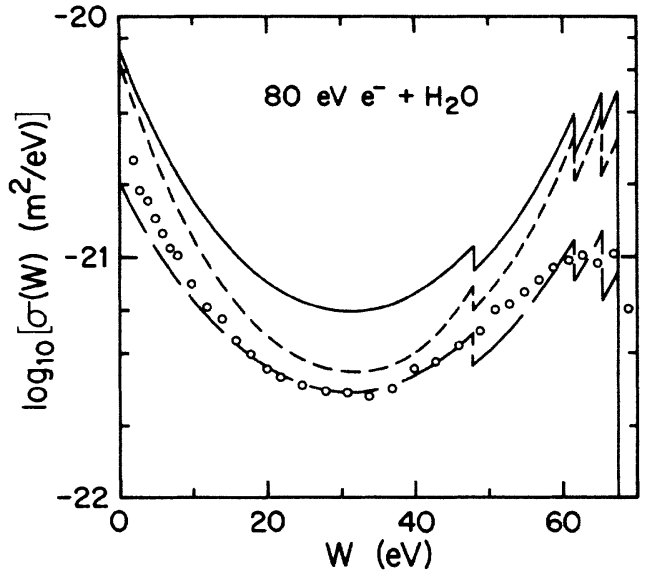

FIG. 4. Energy distribution, integrated over all angles, of electrons from $80-\mathrm{eV}$ collisions of electrons with water vapor. Present data, $\bigcirc$. Theoretical curves, legend same as Fig. 3.

$$
\begin{array}{r}
\sigma(W)=\frac{\sigma_{0}}{T} \sum_{i} N_{i}\left[\frac{1}{\left(W+B_{i}\right)^{2}}+\frac{1}{(T-W)^{2}}\right. \\
\left.-\frac{1}{\left(W+B_{i}\right)(T-W)}\right],
\end{array}
$$

where $\sigma_{0}=4 \pi a_{0}^{2} R^{2}, W$ is the ejected electron energy, $T$ the primary energy, $R$ the Rydberg of energy, $N_{i}$ the number, and $B_{i}$ the binding energy of the $i$ th subshell of the target. Values of $N$ and $B$ used in the calculation are given in Table II. For a single subshell the Mott equation yields a cross-section curve which is symmetric about the energy $W=(T-B) / 2$. However, if more than one binding energy is involved the curve representing the sum over subshells is no longer symmetric. Also since the maximum energy of scattered or ejected electrons is $T-B_{i}$, there are discontinuities in the calculated curve at different energies for different subshells. While there is some minor structure in the measured cross sections there are no sharp discontinuities. This may be due to the superposition of structure due to molecular vibration and other excitations which are not resolved by our analyzer. The Mott formula generally gives results which are too low, especially at high incident energies, but does approximately give the correct general dependence on ejected energy.

Vriens ${ }^{12,13}$ has developed the binary encounter model of collisions applied to electron impact by taking account of the interference between direct and exchange terms. His symmetrical model may be written

TABLE II. Numbers of electrons $N$, binding energies $B$, and ratios of orbital energies to binding energies $G$, for subshells of the water molecule.

\begin{tabular}{llrc}
\hline \hline Subshell & $N$ & $B(\mathrm{eV})$ & $G$ \\
\hline $1 b_{1}$ & 2 & 12.6 & 4.84 \\
$2 a_{1}$ & 2 & 14.7 & 4.02 \\
$1 b_{2}$ & 2 & 18.4 & 2.66 \\
$1 a_{1}$ & 2 & 32.2 & 2.21 \\
$\mathrm{O} 1 s$ & 2 & 539.7 & 1.47 \\
\hline \hline
\end{tabular}




$$
\left.\sigma(W)=\sigma_{0} \sum_{i} \frac{N_{i}}{T+B_{i}\left(1+G_{i}\right)} \mid \frac{1}{\left(W+B_{i}\right)^{2}}+\frac{4 B_{i} G_{i}}{3\left(W+B_{i}\right)^{3}}+\frac{1}{(T-W)^{2}}+\frac{4 B_{i} G_{i}}{3(T-W)^{3}}-\frac{\phi_{i}}{\left(W+B_{i}\right)(T-W)}\right),
$$

where $\phi_{i}=\cos \left\{\left[R /\left(T+B_{i}\right)\right]^{1 / 2} \ln \left(T / B_{i}\right)\right\}$, and $G_{i}$ is the ratio of the orbital kinetic energy to the binding energy of the $i$ th shell. Values of $G$ from Hartree-Fock calculations are given in Table II. Cross sections given by the Vriens equation agree reasonably well with experiment at high incident energies but overestimate the cross section by about a factor of 2 at lower energies.

Gryzinski ${ }^{14}$ has given an equation for the SDCS using the binary encounter approximation in addition to some subsidiary approximations. His results have been integrated over a distribution of orbital velocities. The Fock distribution, which has been used in some binary encounter calculations, ${ }^{15}$ is the quantum-mechanically correct distribution for the hydrogen atom but Gryzinski has used a different distribution. The fact that his results agree less well with experiment at high impact energy than the Vriens equation probably has less to do with his choice of target velocity distribution, however, than with his other approximations. The Gryzinski equation can be written

$$
\begin{aligned}
\sigma(W)=\sigma_{0} \sum_{i} \frac{N_{i} p_{i}}{\left(W+B_{i}\right)^{3}\left(1+p_{i}^{2}\right)^{3 / 2}} & \left\{\frac{W+B_{i}}{G_{i} B_{i}}\left[1-\frac{1}{p_{i}^{2}}\right)+\frac{4}{3} \ln \left[e+\left(\frac{T-W-B_{i}}{G_{i} B_{i}}\right]^{1 / 2}\right]\right. \\
& \left.\times\left(1-\frac{W+B_{i}}{T}\right)^{G_{i} /\left(G_{i}+1+W / B_{i}\right)}\right],
\end{aligned}
$$

where $p_{i}=\left(T / B_{i} G_{i}\right)^{1 / 2}$.

$\mathrm{Kim}^{11}$ has suggested plotting SDCS data on a graph where the ordinate is the cross section divided by the Thomson cross section calculated for the outermost orbital and the abscissa is the energy loss measured in Rydbergs. Thus the quantity

$$
Y=T\left(W+B_{1}\right)^{2} \sigma(W) / \sigma_{0}
$$

is plotted against $(W+12.6) / 13.6$ in Figs. 5 and 6. Dividing by the Rutherford equation eliminates the 4 or 5 order of magnitude range of the cross sections allowing

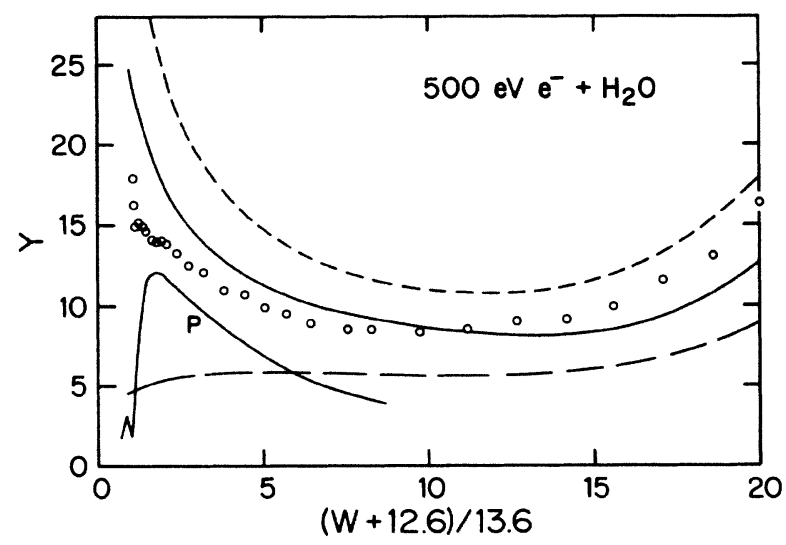

FIG. 5. Energy distribution, integrated over all angles, of electrons from $500-\mathrm{eV}$ collisions of electrons with water vapor. The ordinate is the SDCS divided by the Thomson cross section (see text) and the abscissa is the sum of the ejection energy $W$ and the first ionization potential of water vapor, $12.6 \mathrm{eV}$, divided by the Rydberg energy, $13.6 \mathrm{eV}$. Present data, $O$; theoretical curves, same legend as Fig. 3. $P$ indicates the photoionization data of Samson and Haddad (Ref. 16). them to be plotted on a linear scale. Also, to the extent that the Thomson equation written for the outer subshell is an accurate representation of the overall cross section, one would expect the ratio $Y$ to be a constant equal the number of electrons in the target molecule. At the minimum of the curves in Figs. 5 and 6 the value of $Y$ is indeed in the neighborhood of 10, the expected value.

$\mathrm{Kim}^{11}$ has suggested that while the SDCS at high ejected energies are reasonably well approximated by a binaryencounter calculation such as the Mott equation, the lowenergy region is dominated by distant collisions for which the cross section should be similar to the photoionization cross section. We show the photoionization cross sections measured by Samson and Haddad ${ }^{16}$ in Fig. 5 with an arbitrary ordinate scale. It appears that some combination of that cross section with the Mott cross section would come close to the experimental curve. Miller et al. ${ }^{17}$ and Wilson et al. ${ }^{18}$ have investigated this idea for SDCS by proton impact and have developed an effective algorithm for

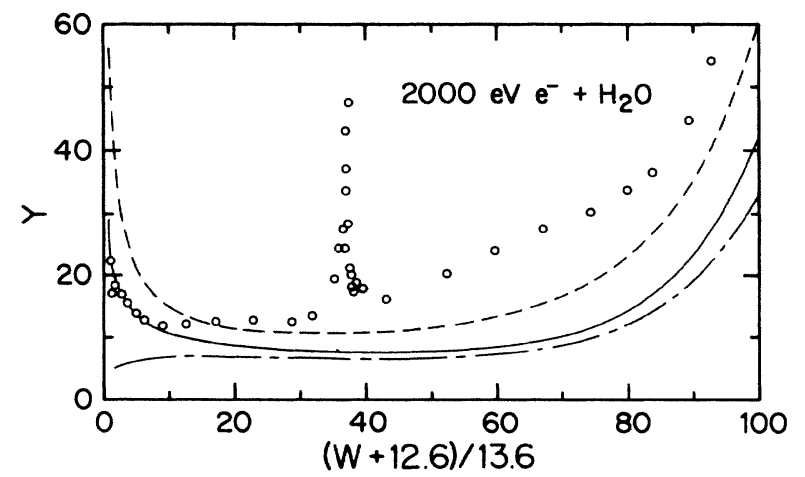

FIG. 6. Same as Fig. 5 for $2000-\mathrm{eV}$ collisions. The peak near $W=500 \mathrm{eV}$ is due to $K$-Auger electrons from oxygen. 
TABLE III. Total ionization cross sections in $10^{-20} \mathrm{~m}^{2}$.

\begin{tabular}{cc}
\hline $\begin{array}{c}\text { Primary energy } \\
(\mathrm{eV})\end{array}$ & Cross section \\
\hline 50 & 2.2 \\
80 & 2.5 \\
100 & 2.5 \\
150 & 2.6 \\
200 & 2.4 \\
250 & 2.1 \\
300 & 1.9 \\
400 & 1.6 \\
500 & 1.3 \\
700 & 1.1 \\
1000 & 0.78 \\
1200 & 0.70 \\
1500 & 0.58 \\
2000 & 0.44 \\
\hline \hline
\end{tabular}

the calculation of SDCS using this approach. They are currently extending it to electron impact.

\section{Total ionization cross sections}

By integrating the SDCS once again, we obtain the total ionization cross sections. One must note, of course, that the scattered primary electron is detected as well as the ones ejected from the target. We have followed the common practice (see, e.g., Refs. 1 and 11) of integrating $W$ from 0 only up to $(T-B) / 2$. When there is more than one shell in the atom or molecule, however, there is no unique value of $B$, the binding energy. Then it is usual to use $B_{1}$, the binding energy of the least tightly bound electron, as an approximation as was done here.

Values of the total ionization cross sections calculated in this way are given in Table III and also plotted in Fig. 7 where they are compared with the more direct measurements of Schutten et al. ${ }^{3}$ and with the proton-impact measurements of Rudd et al. ${ }^{19}$ Since the proton and

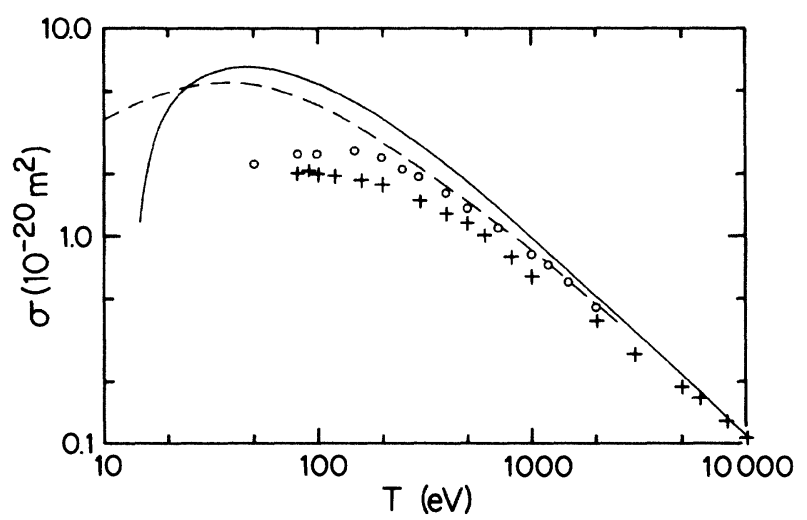

FIG. 7. Total ionization cross sections as a function of impact energy for electrons on water vapor. Present data, $O$; data of Schutten et al. (Ref. 3), +; Vriens equation, solid line; proton-impact cross sections at the same velocity (Ref. 19), dashed line.

equal velocity electron cross sections should approach each other at high energies, the agreement here must be described as excellent, perhaps fortuitously so. However, the present data are 15-35\% higher than those of Schutten et al. ${ }^{3}$ Since both Schutten and we quote a $15 \%$ uncertainty in the measurements, the discrepancy is only slightly outside the combined error bars. Also shown on Fig. 7 is a curve representing the total ionization cross section calculated from the Vriens equation.

\section{ACKNOWLEDGMENTS}

This paper is based on work done under National Science Foundation Grant No. PHY-80-25599. We wish to thank Yong-Ki Kim of the National Bureau of Standards (NBS) for sending us the orbital energies of water vapor and Walter Stevens also of NBS for calculating them. We also wish to thank Kyle Hollman and George Kerby for performing tests on the apparatus.
*Present address: Department of Physics, Shahid Bahonar (Kerman) University, Kerman, Iran.

${ }^{1}$ C. B. Opal, W. K. Peterson, and E. C. Beaty, J. Chem. Phys. 55, 4100 (1971).

${ }^{2}$ D. A. Vroom and R. L. Palmer, J. Chem. Phys. 66, 3720 (1977).

3J. Schutten, F. J. DeHeer, H. R. Moustafa, A. J. H. Boerboom, and J. Kistemaker, J. Chem. Phys. 44, 3924 (1966).

${ }^{4}$ Nobuo Oda, Radiat. Res. 64, 80 (1975).

5J. P. Bromberg, J. Chem. Phys. 50, 3906 (1969).

6M. E. Rudd and R. D. DuBois, Phys. Rev. A 16, 26 (1977).

${ }^{7}$ R. D. DuBois and M. E. Rudd, J. Phys. B 8, 1474 (1975).

${ }^{8}$ H. Ehrhardt, K. H. Hesselbacher, K. Jung, M. Schulz, T. Takaat, and K. Willmann, Z. Phys. 244, 254 (1971).

${ }^{9}$ Sadamu Tahira and Nobuo Oda, J. Phys. Soc. Jpn. 35, 582 (1973).

${ }^{10}$ N. Oda and F. Nishimura, in Abstracts of the Tenth International Conference on the Physics of Electronic and Atomic Col- lisions, Paris, 1977, edited by M. Barat and J. Reinhardt (Commissariat à L'Energie Atomique, Paris, 1977), p. 362.

${ }^{11}$ Yong-Ki Kim, Radiat. Res. 64, 96 (1975).

${ }^{12}$ L. Vriens, Proc. Phys. Soc. (London) 89, 13 (1966).

${ }^{13}$ L. Vriens, in Case Studies in Atomic Physics, edited by M. R. C. McDowell and E. W. McDaniel (North-Holland, Amsterdam, 1969), Vol. I, p. 337.

${ }^{14}$ Michal Gryzinski, Phys. Rev. 138, A322 (1965).

${ }^{15}$ See, e.g., M. E. Rudd, D. Gregoire, and J. B. Crooks, Phys. Rev. A 3, 1635 (1971).

16J. A. R. Samson and G. N. Haddad (private communication).

17J. H. Miller, L. H. Toburen, and Steven T. Manson, Phys. Rev. A 27, 1337 (1983).

${ }^{18}$ W. E. Wilson, J. H. Miller, L. H. Toburen, and Steven T. Manson, J. Chem. Phys. 80, 5631 (1984).

${ }^{19}$ M. E. Rudd, R. D. DuBois, L. H. Toburen, C. A. Ratcliffe, and T. V. Goffe, Phys. Rev. A 28, 3244 (1983). 